\title{
Aspetti internazionali dei problemi delle telecomunicazioni spaziali $(*)$
}

\author{
I. RANZI (**) \\ Ricevuto il 19 Febbraio 1963
}

Riassuxto. - Vengono esposti i problemi che si presentano sul piano internazionale per l'organizzazione dei sistemi di comunicazioni facenti uso di satelliti artificiali della terra come ripetitori, e per lo svolgimento delle ricerche spaziali. In particolare viene trattato il problema dellassegnazione delle frequenze ai vari servizi spaziali e se ne pongono in rilievo le non lievi difficoltà.

Summary. - The paper presents the problems to be tackled on international level for the organisation of communication systems by using artificial satellites of the earth as repeaters and for the performance of space research.

Particular attention is devoted to the problem of the allocation of frequencies to the various space services, emphasizing its considerable difficulties.

Mi propongo di esporre in sintesi quali siano i più importanti problemi tecnici delle telecomunicazioni spaziali in genere, i quali esigono per la loro soluzione una trattazione sul piano internazionale.

Fortunatamente esiste già la sede adatta per tale trattazione, costituita precisamente dall'Unione Internazionale delle Telecomunicazioni (L.I.T.), la cui funzione è fiancheggiata dai suoi due Comitati Consultivi, l'uno per la radiocomunicazione (C.C.I.R.), l'altro per la telegrafia e la telefonia (C.C.I.T.T.). Il grave problema dell'assegnazione delle varie frequenze dello spettro radioelettrico ai diversi servizi viene trattato e

(*) Nota presentata al $2^{\circ}$ Congresso Internazionale Tecnico Scientifico dello Spazio. Roma, 19-23 Gingno 1962.

(**) Professore ordinario presso l'Istituto Superiore delle Poste e Teleco. municazioni - Roma. Presidente della Commissione di Studio per le Comunicazioni Spaziali del Comitato Consultivo Internazionale delle Radiocomunicazioni. 
risolto in tale sede, e si può dire che l'importanza e l'efficienza dell'organizzazione in parola si sono ormai validamente affermate.

Una particolare Commissione di Studio del C.C.I.R., e precisamente la quarta, è stata creata per l'esame dei problemi delle telecomunicazioni spaziali; essa si è riunita per la prima volta a Washington nel Marzo 1962, svolgendo i lavori preparatori per l'Assemblea Plenaria del C.C.I.R. che si terrà a Ginevra nel Gennaio-Febbraio 1963.

Il primo e più importante problema che si presenta in questo nuovo e promettente campo della ricerea e della tecnica è quello dell'assegnazione delle bande di frequenza ai vari servizi spaziali; ad esso, come vedremo, sono strettamente legati in una interdipendenza a volte assai complessa i vari altri problemi teenici dei sistemi di comunicazione spaziale.

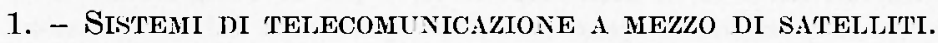

Iniziamo il nostro esame dal campo delle telecomunicazioni a grande distanza, facenti uso di satelliti artificiali della terra, quali ripetitori attivi o passivi.

Le caratteristiche di tali sistemi sono state cosi largamente divulgate in questi ultimi tempi, ed anche nel corso di questo Convegno esse hanno formato oggetto di cosi approfondito esame, che ritengo del tutto inutile ritornare su di esse.

Le più accreditate previsioni che oggi si fanno circa la larghezza totale delle bande di frequenza che verranno richieste dai sistemi in questione sono di $1000 \mathrm{NHHz}$ entro il 1970 e di altri $1000 \mathrm{MIHz}$ entro il 1980; $\dot{e}$ inutile forse precisare che tali cifre si riferiscono ai satelliti ripetitori attivi, gli unici che, allo stato attuale della tecnica, appaiono atti a soddisfare le esigenze di un efficiente istema per comuvicazioni transcontinentali.

Se si confrontano tali esigenze con i $127 \mathrm{MHz}$ complessivi delle varie bande assegnate per tutti i servizi spaziali (comprese quindi le ricerche spaziali) dalla Conferenza Radio Amministrativa di Ginevra del 1959, e se si tiene inoltre conto della completa saturazione dello spettro delle microonde specie fra 1000 e $10.000 \mathrm{NHz}$, il problema sembra a prima vista di soluzione particolarmente difficile.

Fortunatamente, si è ben presto dimostrata la possibilità di condividere le bande richieste con quelle già assegnate ai ponti radio di piccola potenza in visibilità diretta. Sono proprio le frequenze fra 2000 e 10.000 $\mathrm{NHz}$, cioè quelle che comprendono i ponti radio, che appaiono come le 
più adatte per le comunicazioni spaziali; infatti, il limite inferiore a 2000 $\mathrm{MHz}$ o poco al disotto è imposto dal rumore di origine cosmica, la cui intensità cresce rapidamente al disotto di tali frequenze; verso i 4000 $M H z$ l'intensità di tale rumore si riduce a valori inferiori a quelli relativi al rumore dovuto alla emissione di onde elettromagnetiche per mecanismo termico da parte del vapor acqueo atmosferico; anche la radiazione dei corpi posti alla superficie terrestre o quella dello stesso suolo, data la sua intensità relativamente elevata, può contribuire, intorno e al disopra della suddetta frequenza, al rumore del ricevitore, anche se l'antenna non è puntata all'orizzonte, e ciò per effetto dei cosidetti lobi secondari dell'antenna stessa.

Il rumore dovuto al vapor acqueo atmosferico s'accresce poi con la frequenza al disopra di quella banda intorno ai $4000 \mathrm{MHz}$, a cui corrisponde cosi il minimo del rumore di origine esterna; si progetta pertanto di assegnare tale banda (precisamente fra 3700 e $4200 \mathrm{MHz}$ ) alle comunicazioni dirette dal satellite verso terra, tenuto conto della limitata potenza dei trasmettitori posti a bordo dei satelliti, e della necessità quindi dell'impiego di sistemi riceventi a terra di elevata sensibilità.

İ poi previsto l'uso delle due bande fra 1750 e $2250 \mathrm{MHz}$ e fra 5925 e $6425 \mathrm{MHz}$ per le trasmissioni dalle stazioni a terra verso i satelliti; un'altra banda fra 6500 e $7000 \mathrm{MH} z$ potrà essere necessaria per gli ulteriori sviluppi dei sistemi di telecomunicazioni a mezzo di satelliti (verso il 1980).

In linea di massima, tutti i Paesi occidentali appaiono d'accordo su tali assegnazioni; nella riunione di Washington, la delegazione dell'Unione Sovietica ha espresso il parere che sia per ora sufficiente assegnare ai servizi in questione solo tre bande, una di 100, una di $400 \mathrm{e}$ una di $500 \mathrm{MHz}$, fra 3000 e $6000 \mathrm{MHz}$. La decisione finale spetterà alla Conferenza Radio Amministrativa prevista per la fine del 1963, a Ginevra.

La condivisione delle frequenze con i ponti radio presenta vari problemi, i cui aspetti hanno una diretta importanza nel campo internazionale.

La possibilità di interferenze reciproche fra $\mathrm{i}$ due sistemi va attentamente esaminata. Praticamente non avverrà mai che l'antenna a terra di un sistema di comunicazione a mezzo di satelliti e quella di un terminale di un ponte radio siano puntate direttamente l'una verso l'altra; tuttavia l'esistenza dei lobi secondari delle stesse antenne, specie dei primi lobi laterali, può far sì che parte dell'energia emessa da un'antenna entri nell'altra, anche se gli angoli fra gli assi siano dell'ordine di una diecina di gradi o più. Nel caso della interferenza prodotta in un ricevitore spaziale da parte delle emissioni di un ponte radio, è l'elevata sen- 
sibilità del ricevitore a definire le condizioni di protezione, e nel caso della interferenza inversa, è l'elevata potenza del trasmettitore del sistema spaziale (dell'ordine di uno o più chilowatt) a definire i termini del problema.

E ovvio che il valore limite massimo consentito, per quanto riguarda la potenza del segnale disturbante all'ingresso del ricevitore, dipende in alto grado dai tipi di modulazione del segnale desiderato e del segnale disturbante: nel caso di trasmissioni di telefonia, comprendenti un certo numero di canali (ad esempio, 960 canali a due vie, in una banda base di $5 \mathrm{MHz}$ ), appare subito la convenienza di disporre nella stessa banda di frequenza, i vari canali dei due sistemi di comunicazione, in modo tale che quelli di un sistema siano intercalati fra $\mathrm{i}$ singoli canali dall'altro sistema.

Poichè esistono dei precisi piani di canalizzazione raccomandati in sede internazionale dal C.C.I.R. per i ponti radio, sarebbe possibile una riduzione delle probabilità di interferenze adottando un opportuno piano di canalizzazione per i sistemi a saltellite; vi sono, tuttavia, oggi alcune difficoltà per un tale accordo, dato che per alcune bande di frequenza vi sono Paesi che, per ragioni tecniche ed anche finanziarie, non sono in grado di modificare i loro impianti di penti radio, così da adeguarsi alle norme del C.C.I.R. emanate dopo la messa in opera degli impianti stessi.

Anche quando sia possibile una disposizione interlacciata dei canali, la protezione dalle interferenze può essere assicurata solo se viene interposta fra le stazioni terminali dei due sistemi una notevole attenuazione di propagazione; questa attenuazione appare dell'ordine di 160 dB e doviebbe salire a $180 \mathrm{~dB}$ circa nel caso in cui l'interlacciamento non sia possibile.

Attenuazioni di quest'ordine si possono avere nella propagazione troposferica al di là dell'orizzonte, a mezzo del noto meccanismo della diffusione (scatter), oppure nel caso dell'interposizione di rilievi del terreno, quali catene montuose, che producano una efficiente azione di schermo; quest'ultimo effetto è oggi assai poco noto nei suoi dettagli e intense ricerche in tale campo sono state raccomandate dal C.C.I.R.

Le distanze cui corrispondono le richieste attenuazioni di propagazione vanno dai 40 ai 150 e più chilometri; è ovvio come, anche nella previsione del caso di terminali dei due sistemi posti vicino a frontiere fra due Stati, sia necessaria una trattazione del problema in sede internazionale.

Il problema, comunque, va trattato tenendo anche presente il fatto che le stazioni a terra dei sistemi di comunicazioni spaziali saranno in 
numero limitato su tutta la terra e che, quindi, una volta fissati i criteri generali per la soluzione, le soluzioni singole andranno studiate caso per caso. Soluzioni di carattere generale non possono ora essere date, anche perchè i sistemi di modulazione più convenienti per le comunicazioni a mezzo di satelliti saramno definiti solo in base ai risultati delle esperienze in corso col satellite "Telstar" e delle varie altre progettate (satelliti "Relay " e "Syncom ", in particolare).

Non solo il tipo di modulazione, ma anche la potenza in gioco, tutti clementi questi che influiscono sulle possibilità di interferenze con altri sistemi, dipendono dal sistema a satellite che verrà in definitiva adottato. Allo stato attuale delle nostre conoscenze, sembra che i sistemi suscettibili di pratiche applicazioni nel campo delle telecomunicazioni siano i seguenti:

a) sistema con numerosi satelliti, distribuiti casualmente, in orbite ellittiche o rircolari inclinate sul piano equatoriale, ad altezze da 2000 a $5000 \mathrm{~km}$ (irca sulla superficie terrestre (tipo "Telstar );

b) sistemi con 12 o 20 satelliti in orbita circolare equatoriale ad altezza intermedia (intomo ai $12.000 \mathrm{~km}$ ), dotati di dispositivi per la correzione dell'orbita e della velocità, cosi da mantenere l'equidistanza fra di loro, e dotati inoltre di dispositivi automatici per l'orientamento fisso verso terra così da consentire l'uso di antenne direttive a elevato guadagno;

c) sistemi con satelliti sincroni col moto della terra, su orbita equatoriale (alta cirea $36.000 \mathrm{~km}$ ) e quindi stazionari rispetto alla superficie terrestre, dotati di dispositivi per la correzione dell'orbita e per l'orientamento fisso (3 di tali satelliti sarebbero sufficienti).

E evidente che nel caso del sistema $b$ ), in cui le orbite dei satelliti sono sempre le stesse per una determinata stazione e ancor più nel caso del sistema stazionario, in cui le antenne delle stazioni a terra mantengono un puntamento fisso, le possibilità di interferenza con ponti radio sono più ridotte.

Nel caso del sistema con satelliti stazionari vi è, però, da tener presente che forse il tipo di modulazione più conveniente nelle trasmissioni dalla terra verso il satellite potrà essere quello a bancla laterale unica; esso, infatti, consentirebbe una magrorio accessibilità al sistema da parte delle stazioni a terra.

Come già si è detto, solo l'esperienza fornirà gli elementi per la scelta del sistema; non è, però, da escludersi che risulti conveniente l'impiego simultaneo di due diversi sistemi. 
Sempre per ciò che riguarda il problema della condivisione delle frequenze, sono oggetto di studio anche le possibilità di interferenza nei ricevitori dei ponti radio per effetto delle emissioni dai satelliti, e quella di interferenza nei ricevitori a bordo dei satelliti da parte di trasmettitori di ponti radio.

Gli studi nel campo della condivisione delle frequenze mirano ora a stabilire le condizioni generali e i modi di soluzione dei vari problemi, che potranno in seguito delinearsi con maggiore precisione.

Oltre alle bande di frequenze destinate alle comunicazioni vere e proprie (telefoniche o televisive), altre più ristrette bande sono richieste dai satelliti per comunicazioni: una banda per il puntamento e l'inseguimento (tracking) del satellite, e per la trasmissione dei dati sul funzionamento degli apparati di bordo, sulle radiazioni ambientali, ecc. (telemetria), banda che già è stata assegnata, sia pure non in esclusiva, dalla Conferenza di Ginevra del 1959, fra 136 e $138 \mathrm{MH} z$; un'altra banda, di pochi $\mathrm{KH} z$, attorno ai $400 \mathrm{MHz}$ sarà necessaria per la trasmissione di comandi da terra (telecomandi), comandi relativi all'entrata in funzione dei vari apparati o al loro silenziamento, all'eventuale funzionamento di dispositivi per la correzione dell'orientamento o del noto del satellite, ecc.

$\mathrm{Vi}$ sono altri problemi relativi all'entrata in funzione di un sistema di comunieazioni intercontinentali a mezzo di satelliti, problemi che esigono una discussione in sede internazionale; ci limiteremo qui ad enunciarli:

a) Diffusione dei dati orbitali dei vari satelliti così da consentire il puntamento delle antenne delle stazioni a terra.

b) Assegnazione dei canali telefonici e televisivi ai vari Paesi.

c) Dislocazione delle stazioni a terra, e interferenze fra stazioni vicine.

d) Metodi per la commutazione da un satellite all'altro, specie nei sistemi a satelliti distribuiti casualmente in orbite relativamente basse.

e) Metodi per la correzione dell'effetto Doppler.

f) Interconnessioni fra stazioni a terra dei sistemi a satelliti e sistemi di comunicazioni terrestri.

\section{2. - TELECOMUNICAZIONI SPAZIALI IN GENERE.}

Anche in questo campo, il problema fondamentale che si presenta sul piano internazionale è quello dell'assegnazione delle frequenze. Considereremo qui brevemente quali siano le esigenze nei vari casi. 
2.1 Sonde spaziali (tipo "Ranger", "Maviner », ecc.). - Per quanto riguarda il "tracking " normale potrà servire la stessa banda 136-138 $\mathrm{NHz}$ già assegnata; il tracking di precisione che tali sonde esigono dovrà farsi su frequenze più alte fra 1000 e $4000 \mathrm{MHz}$ e con elevate larghezze di banda (da 2 a $4 \mathrm{MHz}$ ), dato che si deve determinare con grande esattezza anche la distanza, e si deve far quindi uso di emissione e ricezione di impulsi (transponder); tali frequenze potranno essere condivise fra le varie sonde spaziali se queste sono assai lontane fra di loro. I ata, però, la piccola potenza dei segnali ricevuti da sonde lontane, occorrerebbe provvedere all'assegnazione di almeno una banda in uso esclusivo per l'esplorazione profonda dello spazio.

La telemetria e il telecomando potrebbero elfettuarsi fra 1000 e $4000 \mathrm{MHz}$, o, come proposto da taluni, secondo due bande, l'una fra 300 e $600 \mathrm{MIHz}$, e l'altra fra 600 e $2000 \mathrm{MHz}$, per una larghezza totale di $1 \mathrm{MHz}$. La prima banda appare come la più conveniente, poichè consente l'impiego, a bordo della sonda, di trasmettitori a transistor, di peso e ingombro assai ridotto.

2.2. Satelliti per riccrche. - Anche per questi satelliti la banda fra 136 e $138 \mathrm{MHz}$ viene proposta per una definitiva assegnazione alle funzioni di tracking e telemetria. Per il tracking di precisione, per la trasmissione di dati a larga banda e per particolari ricerche su fenomeni di propagazione, si prevede l'impiego di frequenze anche più elevate, sino a $10 \mathrm{GHz}$.

L'Unione Sovietica tende a impiegare onde H.F., fra i 20 e i $90 \mathrm{MHz}$; le frequenze più basse offrono il vantaggio di poter essere ricevute, in seguito a riflessioni multiple fra la ionosfera e la superficie terrestre, anche quando il satellite si trova occultato dalla terra, il che può essere utile quando non si possa disporre di una rete mondiale di stazioni di ascolto; tali frequenze offrono però lo svantaggio di un elevato rumore di origine atmosferica e cosmica e della possibilità di interferenze.

2.3 Satelliti di navigazione. - Per la telemetria e il telecomando vengono proposte le bande di frequenza già indicate per gli altri satelliti e per le sonde spaziali. Le frequenze, su cui verranno effettuati i rilievi che conducono alla determinazione della posizione di chi riceve i segnali, variano a seconda del sistema usato. Come è noto, in tutti i sistemi si presumono conosciuti tutti i parametri dell'orbita del satellite. In quei sistemi in cui il punto viene fatto misurando l'angolo d'arrivo verticale 
(oppure quello orizzontale) dei segnali emessi dal satellite, le frequenze dovranno essere nella gamma fra 8 e $35 \mathrm{GHz}$. Nei sistemi basati invece sul metodo del "transponder") (emissione di impulsi del satellite, su comando da terra, e determinazione del tempo di percorso), la frequenza dovrà rimanere fra i 300 e i $700 \mathrm{MHz}$. Nei sistemi, infine, basati sulla misura dell'effetto Doppler nei segnali ricevuti, le frequenze dovranno preferibilmente cadere fra 100 e $1000 \mathrm{MHz}$.

2.4 Satelliti meteorologici. - Il successo dei vari satelliti meteorologici della serie "Tiros " lanciati dagli Stati Uniti, la preparazione della nuova serie dei satelliti "Nimbus ", rendono di grande importanza, per lo sviluppo della meteorologia su scala mondiale, l'assegnazione di adeguate bande di frequenza per tali ricerche.

Mentre per il tracking può bastare la consueta banda fra 136 e $138 \mathrm{MHz}$, la trasmissione dei dati (in particolare delle fotografie delle nubi) dovrebbe avvenire nella banda, sino ad oggi usata, fra 1700 e 1710; questa banda fu già assegnata dalla conferenza di Ginevra del 1959, ma non in uso esclusivo.

Si progetta ora di dotare tali satelliti di piccoli radar per l'osservazione delle nubi (su frequenze intorno ai $35 \mathrm{GHz}$ ) e delle precipitazioni (su frequenze vicine a $10 \mathrm{GHz}$ ); ̀̀ prevista anche l'emissione di microonde di frequenze corrispondenti ai massimi di assorbimento da parte del vapor acqueo (23 GHz) e dell'ossigeno atmosferico (60 GHz), ciò al fine di studiare la distribuzione di tali componenti atmosferici.

2.5 Veicoli spaziali pilotati. - Te comunicazioni fra veicoli spaziali pilotati e le stazioni a terra comprendono, oltre al collegamento telefonico a due vie, la trasmissione dei dati di comando, controllo, guida, navigazione, la trasmissione televisiva, e inoltre uno speciale collegamento per i casi di emergenza.

Si prevede che la fase sperimentale dei voli pilotati continuerà per almeno dieci amni, e in questa fase, mentre alcume funzioni (come il tracking, i controlli, ecc.) potrammo essere svolte sui canali già proposti per i veicoli pilotati, occorre disporre di una banda per comunicazioni radiotelefoniche sotto i $25 \mathrm{MHz}$ (cosi da assicurare la riduzione anche al di là dell'orizzonte visibile dal veicolo), e di una banda fi"a 100 e $600 \mathrm{MMHz}$, avente una larghezza di circa 0,5 MHz, e con una elevatissima protezione dalle interferenze; praticamente, tale banda dovrebbe essere assegnata in uso esclusivo, dato che, in condizioni di emergenza, la vita del pilota dipende soltanto dalla possibilità di trasnettere al pilota istruzioni o comandi diretti da terra al veicolo stesso. 
Specialmente nella fase di rient ro le condizioni dei radiocollegamenti si famno assai difficili, a causa della formazione di uno strato di "plasma " attorno al veicolo, ossia di nono strato di gass atmosferici fortemente ionizzati a causal del calore che si svolge per l'attrito incontrato dal veicolo nel suo lientro nell'atmosfera; soltanto onde di fiecquenza assai elevata, forse superiore ai $10 \mathrm{GHz}$, potranno sorpassane questo strato senza subire un alssorbimento troppo alevato, ed è appunto in questa gatmma di frequenze che occorrerà asseggnare una delle bande di emergenza per le comunicazioni ron i veicoli spaziali pilotati.

2.6 Radio diffusione diretta dai satelliti. - Allo stato attuale, non si preverle certo prossina la realizzazione di emissioni di radio diffusione da satelliti con potenza tali che la ricezione a terra possa avvenire con i nomali apparecechi di uso domestico. Quando si pensi che occorrerebbero trasmettitori a bordo dei satelliti con potenze effettive irradiate che nel ampo delle V.H.F. dovebbero superare i $100 \mathrm{~kW}$, e si tiene presente che le potenze massime emesse ora dai satelliti sono dell'ordine di una diecina di watt, si vede quali difficolta si incontrino in tale campo. E (iò senza tener conto delle non meno lievi difficoltà circa la scelta di programmi che devono interessare e risultare graditi ad assiemi di popolazioni diverse per lingua es struttura politica.

9.7 Radio Astronomia. -- L'impiego di sistemi riceventi di elevatissina sensibilità, dotati in genere di dispositivi di integrazione dei segnali nel tempo, rende assai difficile il problema della scelta delle frequenze per le osservazioni di radio astronomia, sia che si tatti della ricezione delle radioonde emesse da sorgenti cosmiche, sia che si tratti della ricezione delle onde dirette da trasmettitori terrestri su corpi celesti, a da questi retrodiffuse verso terra (Astronomia "Radar"); in quest'ultimo caso, l'elevatissima potenzal dei trasmettitori pone il problema della interferenza nei sistemi di communicazione terrestri.

La Conferenza di Ginevia del 1959 aveva raceomantato la protezione di alcume bande di frequenza, e in particolare di quella relativa alla emissione da parte dell'idrogeno cosmico (1420-1425) $\mathrm{NHz}$; in pratica, tuttavia, l'elevato livello delle interferenze rende in molti Paesi assai difficili le osservazioni radioastronomiche.

La condivisione delle frequenze con altri servizi, specie nella banda U.H.F., appare assai difficile, a meno di non installare gli osservatori in località assai lontane dai trasmettitori dei servizi terrestri, e protette da catene montuose. In alcuni casi si può pensare a una condivisione 
nel tempo, ossia a una sospensione in certi periodi della giornata delle emissioni dannose per le osservazioni radioastronomiche.

\section{ConClusione.}

Il quadro sommario che si è cercato di dare delle esigenze dei vari sistemi di commicazione connessi con le ricerche e le applicazioni delle nuove tecniche spaziali puó dare un'idea della complessità dei problemi che sorgono sul piano internazionale, specie per quanto riguarda l'assegnazione delle frequenze e l'eliminazione delle interferenze.

In particolare, i contrasti fra le esigenze delle ricerche, specie di quelle radio astronomiche, a la pressante richiesta di bande di frequenze per servizi terestri di estrema importanza sia nel ampo civile che in quello n!ilitare, danno luogo a probleni che a volte sembrano insolubili.

Mi sia consentito di formulare il roto che si trovi la possibilità di assicurare lo svolgersi anche di quelle ricerche che, pur non offrendo ora alcuna possibilità di pratica applicazione, hamno tuttavia il nobile intento di approfondire le nostre conoscenze sull'universo che ci circonda e di elevare il nostro spirito nella contemplazione delle meraviglie che l'miverso stesso racchinde. 